\title{
Home Automation using EMOTIV: Controlling TV by Brainwaves
}

\author{
Marquos Zaki*, Ali Alquraini**, Tarek Sheltami \\ King Fahd University of Petroleum and Minerals, Dhahran, Saudi Arabia, 31261
}

\begin{abstract}
In this paper, our goal is to prove the possibility of controlling a home device by solely using neural pattern recognition interface captured by Emotiv EPOC. The neuroheadset EPOC is a personal interface for human interaction with computer through the acquisition of electrical signals produced by the brain, via techniques of electroencephalography (EEG), in order to identify thoughts, feelings and facial expressions in real time. The pattern is then fed to a client, which communicates it to the server side where it is received by a Raspberry Pi microcontroller. The microcontroller sends the appropriate response based on what is received from the client, which turns on or off a TV. This application will have a great potential especially for people with Amyotrophic Lateral Sclerosis (ALS) or special needs in general.
\end{abstract}

Keywords: EEG, EMOTIV, Home Automation, eHealth, RasperryPi, BCI

\section{Introduction}

Electroencephalograms (EEG) are commonly used by in hospitals and health related institutions to enable doctors monitor and record the electrical activity of the brain. This enables them to detect brain activity and potential abnormal patterns, which gives them good insight on the overall brain health and helps to diagnose brain related diseases. In this study, an EEG-based brain computer system is proposed for automating home appliances. Brain Computer Interface (BCI) is a system that provides direct pathway between the brain and external computing resource or devices. Recent development of commercial EEG devices offers an affordable solution to explore and develop applications in various domains. A particularly promising study has proved the ability to reach more than $75 \%$ recognition accuracy using some of those device [1], which makes it highly desirable for control tasks.

For this reason, we have chosen EMOTIV EPOC+ as our telemetric EEG device for data acquisition as seen in Fig.1. EEG device has 14 channels (electrodes) with sampling frequency of $128 \mathrm{~Hz}\left(2048 \mathrm{~Hz}\right.$ internal). It has a built-in $5^{\text {th }}$ order low pass sin filter of bandwidth 0.2 to $45 \mathrm{~Hz}$, and connected wirelessly to the computer through a $2.4 \mathrm{GHz}$ band [2].

Fig.1 shows the Emotiv EPOC headset and the location of the electrodes relative to the $10 / 20$ system positioning.

\footnotetext{
* Corresponding author. Tel.: +966531934874

E-mail: morqus.samuel@gmail.com

*** Author. Tel.: +966599833339

E-mail: ali.h.alquraini@gmail.com

(C) 2018 International Association for Sharing Knowledge and Sustainability.

DOI: $10.5383 / J U S P N .10 .01 .004$
}

We use an efficient system to control home appliances (TV in our case). The system is composed of three units: EMOTIV headset, a personal computer and Raspberry Pi 3 [3]. Our aim is to achieve our goal using a simple, low cost and straightforward method without the need for a special software or extensive development.

\section{Related Work}

The idea of proposing environmental control system is not new, several systems were proposed for people with disabilities to control their surroundings[4]. One approach uses Radio frequency identification (RFID) [5], others enhanced it with voice control [6]. While these systems work well with motiondisabled people, they do not serve people with vision or voice impairments. On the other hand, EMOTIV EPOC, despite of being relatively new in EEG market, uses Brain- Computer Interface (BCI) technology to manipulate digital content wirelessly. It is capable of capturing facial gestures and expressions along with mapping brain patterns to various mental commands such as; pushing, pulling, rotate left and rotate right. Several projects use telemetric EEG headsets devices in order to facilitate the interaction between physically challenged and their environment [7].

In [8] an attempt of designing of BCI controlled chair using Arduino platform to receive the directions from the headset and controlling servomotors, thus moving the chair. However, a core limitation is the assumption of a permanently wired 
connection from laptop to the Arduino. A similar scenario to to our system is presented in [9] by controlling an array of LEDs using Arduino. Although it did show that controlling LEDs with EMOTIV is possible, the authors connected the device to the computer locally and did not propose a method for controlling the LEDs using the headset over the internet. Thus a valuable potential of the device was not utilized. Little work exist that combines both the potential of an EEG headset and an adequate wireless infrastructure. In [10], the authors built a platform that uses IoT paradigms in connecting EEG headsets with home devices using the cloud. Other extensive work in [11] proposed a system for controlling home applications using EMOTIV EPOC and embedded module. A third interesting approach is demonstrated in [12], where the authors used Neuroky headset for signal acquisition connecting it to an Arduino using a Bluetooth module. The Arduino controls a DC motor and a light bulb simulating different home appliances. They included a simple URL for monitoring the devices in use. In [13], the authors have developed a virtual home environment that can be controlled using brainwaves, however they did not implement it in a real life environment. The work presented here demonstrates the use of brainwaves in controlling a real physical device with the assistance of EMOTIV EPOC headset.

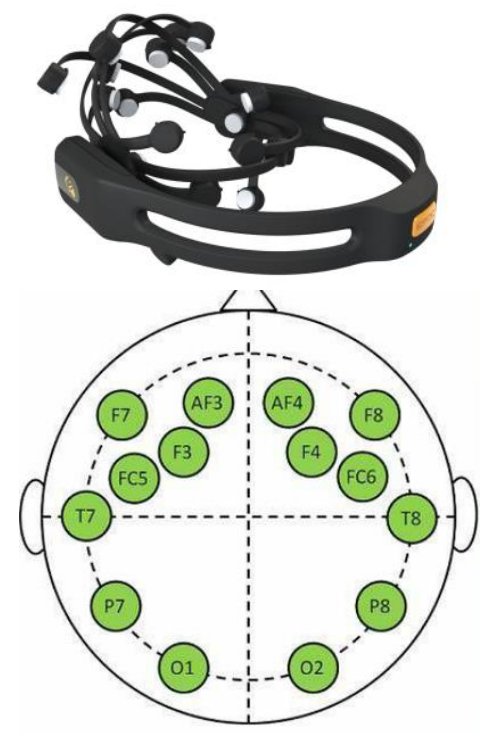

Fig. 1. EMOTIV EPOC+ headset and electrodes location

\section{System Description}

In this section, we describe our system and highlight all required software and hardware in our implementation.

\subsection{System Architecture}

The system architecture (Fig. 3) consists of three main stages starting with data acquisition, data transmission and finally device controlling. First, the Emotiv EPOC+ headset reads and collects the signal pattern from the brain and sends this data to a computer using EmoEngine, which is an application developed by EMOTIV to handle decoding, processing and mapping them to the trained patterns of the user. EMOTIV provides a Control Panel, depicted in Fig. 4, to interface with EmoEngine. All subjects are trained with the control panel to control pull movement of the virtual cube and clench gesture.
Those patterns could be detected by Emokey (Fig. 6), another suite provided by Emotiv for mapping thought patterns to keyboard input. For example, in our case we implement a condition to send " 0 " or " 1 " for turning off or on devices that we would like to control. Second, the computer acts as a client as seen in Fig. 7 and initiates a connection to a server using a simple socket script written in Python. Figure 2 shows the flowchart of the client python script.

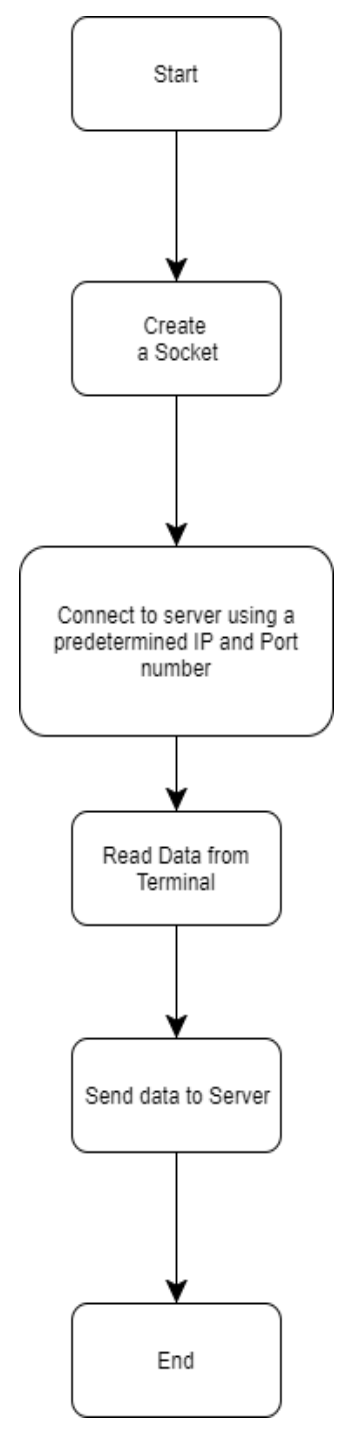

Fig. 2. Client script flowchart

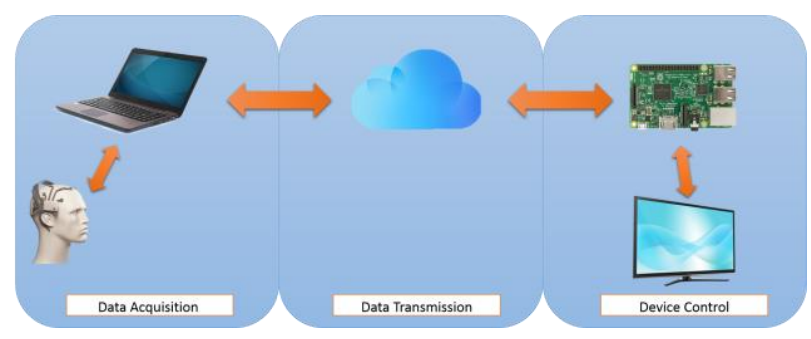

Fig. 3. System Architecture Overview 


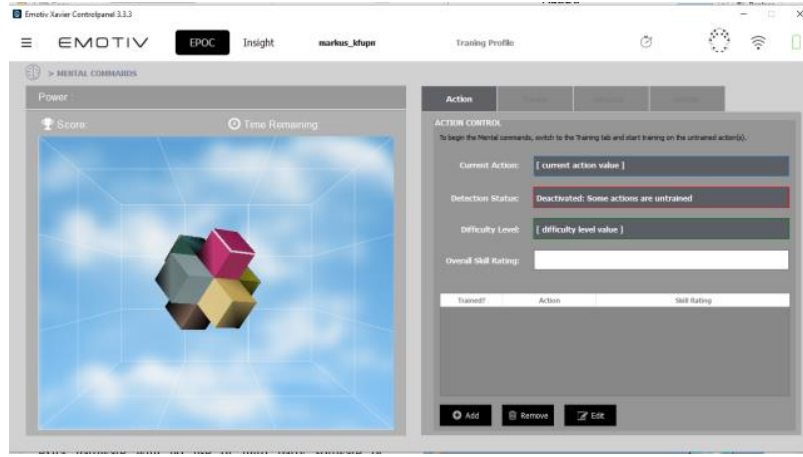

Fig. 4. Training module, Emotive Control Panel

Using a client-server protocol to establish a TCP connection, the client sends these data that are received from the headset to the server. Finally, on the other side of the socket, a server is running on Raspberry Pi listening to a dedicated port (Fig. 8) is awaiting for a request, once it receives the request, the connection is established and the data is received by the Raspberry Pi. It then sends a signal to turn the device ON or $\mathrm{OFF}$, which is a TV in our case. Figure 5 shows the flowchart of the python script used as a server.

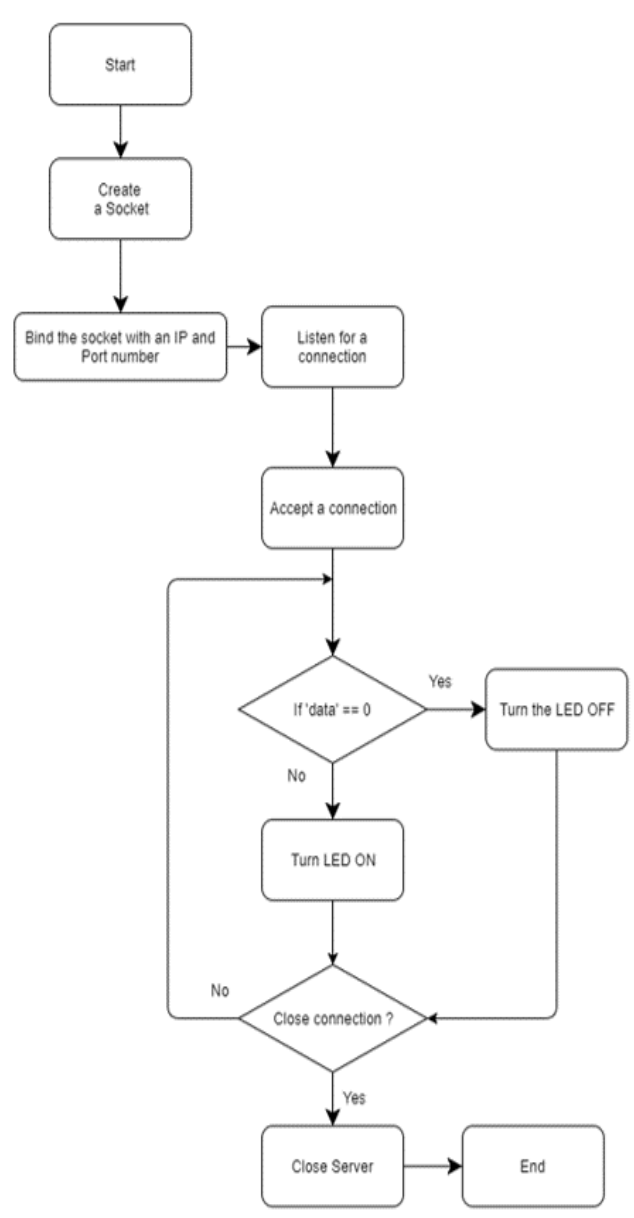

Fig. 5. Server Code flowchart

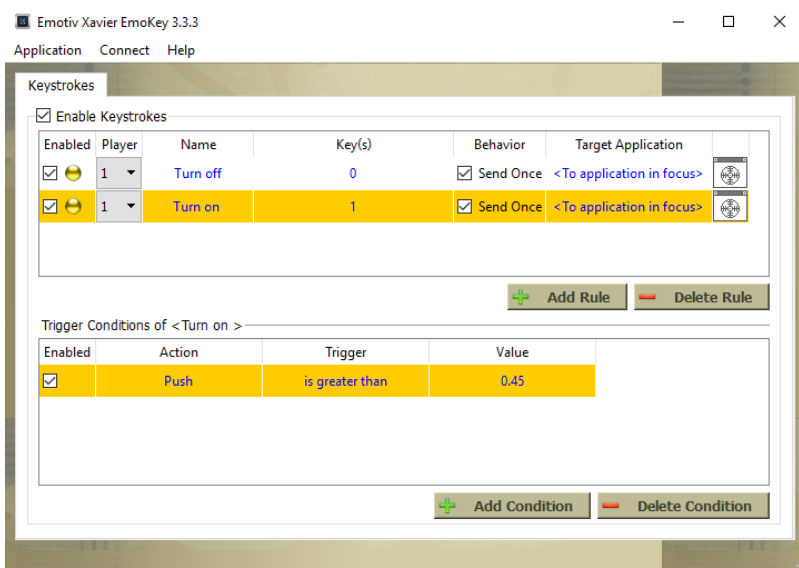

Fig. 6. Control Panel, Emokey

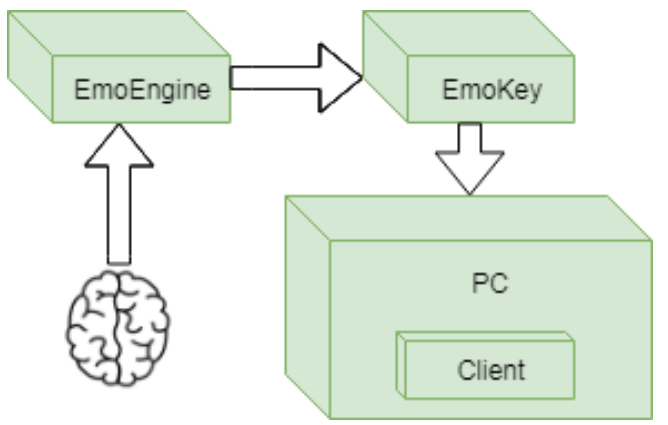

Fig. 7. System Architecture, Data Acquisition.

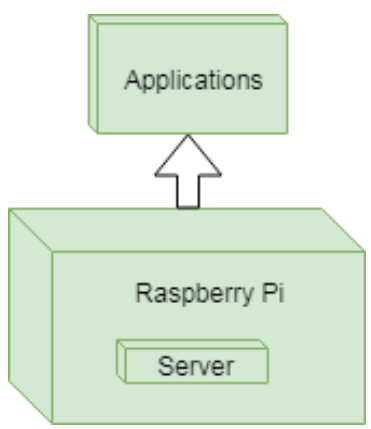

Fig. 8. System Architecture, Receiver Side block

\subsection{Design Flow}

A flow chart is a graphical representation of the logic sequence of the system flow is shown in Fig. 9.

The diagram shows how a home appliance could be controlled using brain signals, which are transmitted over the internet using a simple TCP connection. It proceeds as follows; the first 
step is to initialize EPOC headset and connect it to a personal computer or a laptop, which is achieved using one of two ways supported by the headset, namely Bluetooth or using Wi-Fi dongle that accompanies it. The next step is to launch Emotiv Control panel (Fig. 4) and make sure its connected to EmoKey (Fig. 6) and correctly reading the headset sensors. Next, the python script connects to the server. The server is running on Raspberry Pi waits for the received signal. Lastly, the server checks if the received character is 1 or 0 . Depending on the received signal, the Raspberry Pi sends the corresponding signal to the infrared transmitter, which turns the TV ON or $\mathrm{OFF}$

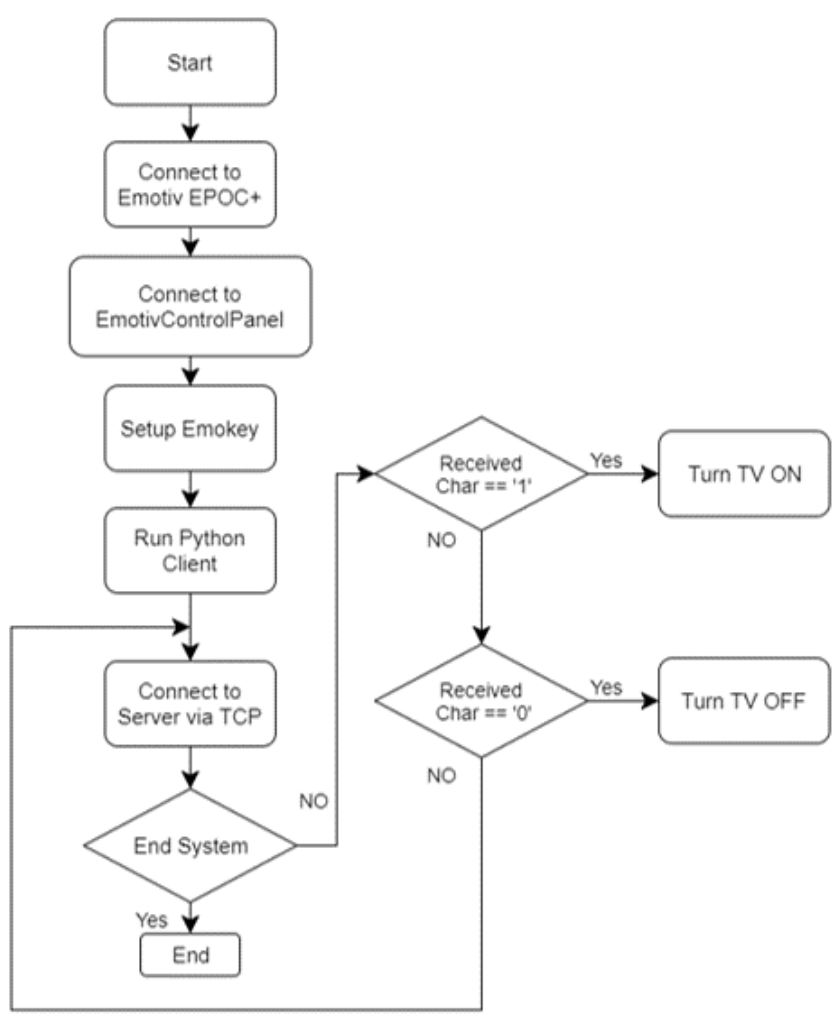

Fig. 9 System Flowchart

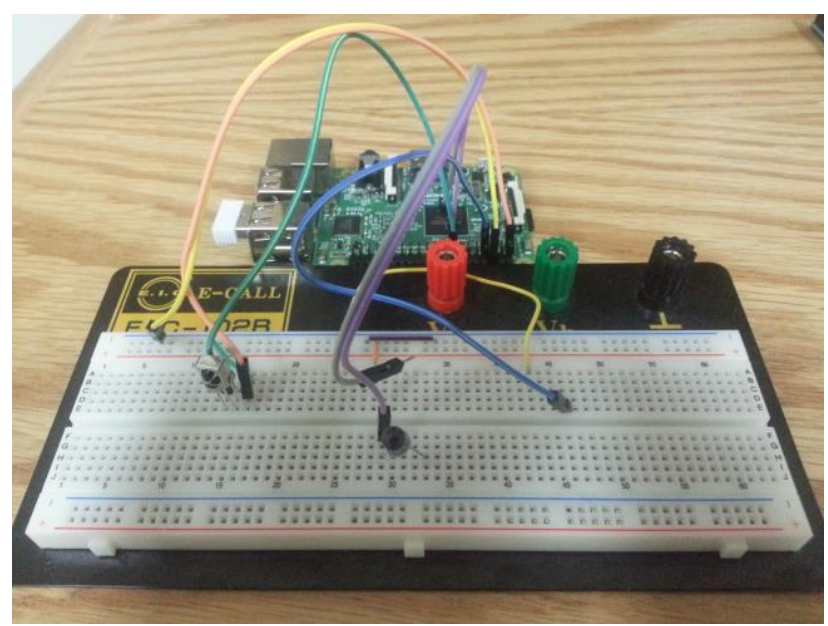

Fig. 10 a circuit showing Raspberry PI connected to infrared transmitter and receiver.

\section{Results and Discussion}

The experiment was conducted using brainwaves acquired from a healthy male subject. The research edition SDK of EMOTIV EPOC, in addition to the available software provided by EMOTIV, supplied the EMOTIV Pro control panel to allowed real time monitoring to the raw data read from the 14 sensors as shown in Fig. 11.

The subject is trained using the Emotiv Control Panel (Fig. 4) to control the push movement and the teeth clench movement. The push movement is mapped by Emokey (Fig. 4) to send ' 0 ' and the clench to send ' 1 '. The raw data of both movements is shown in Fig. 12, a and b.

We used EmoComposer (Fig.13) provided with EMOTIV suite to do the initial testing. It allows running a script with manually entered values for each sensor. This enabled adjusting the sensitivity of each cognitive action and monitor the headset response in a controlled simulation environment.

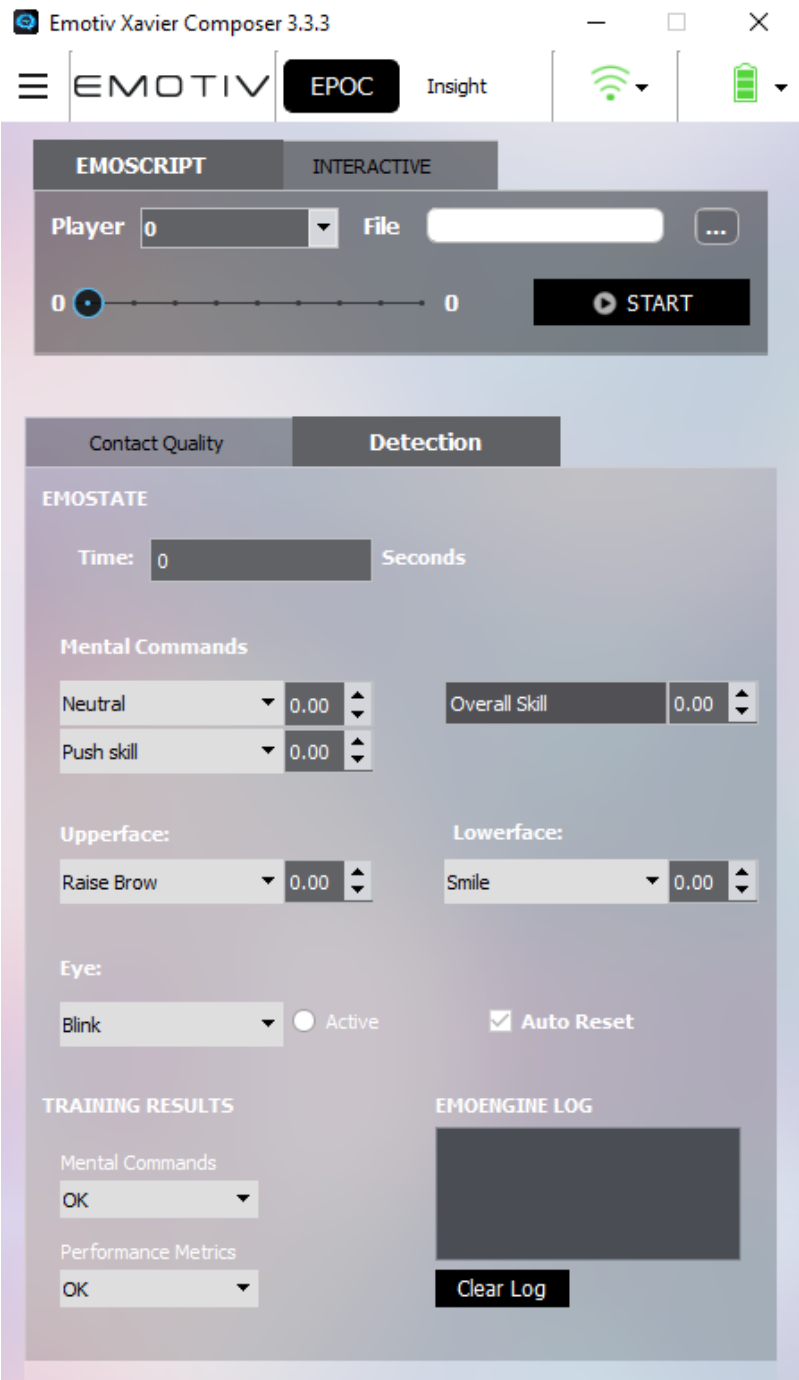

Fig. 13 EmoComposer window 


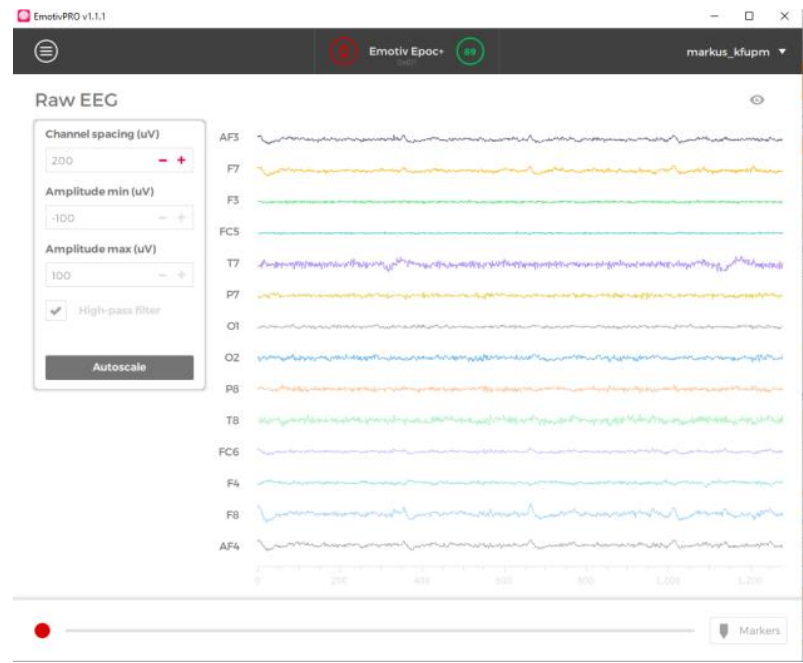

Fig. 11 Real time EEG readings corresponding to the raw data read by the 14 channels.

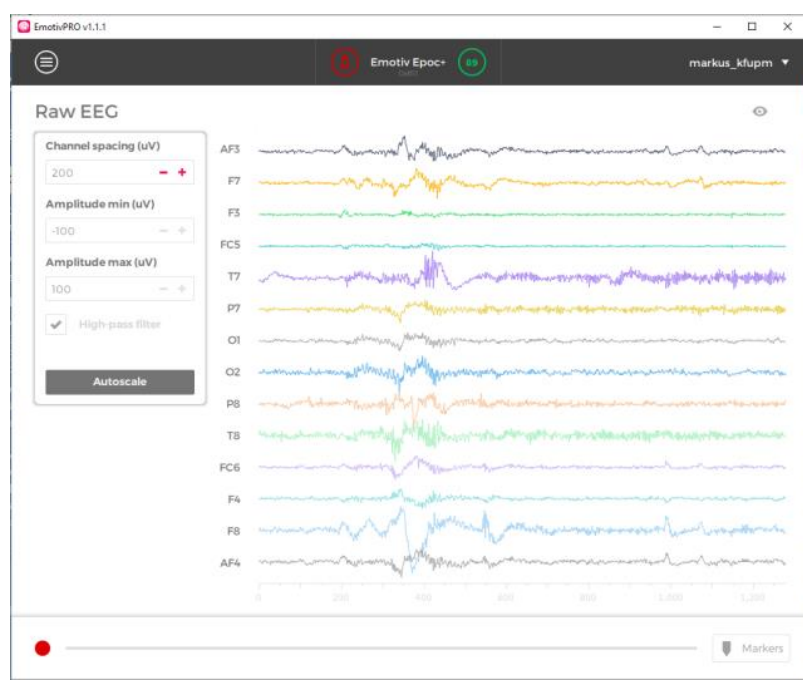

(a)

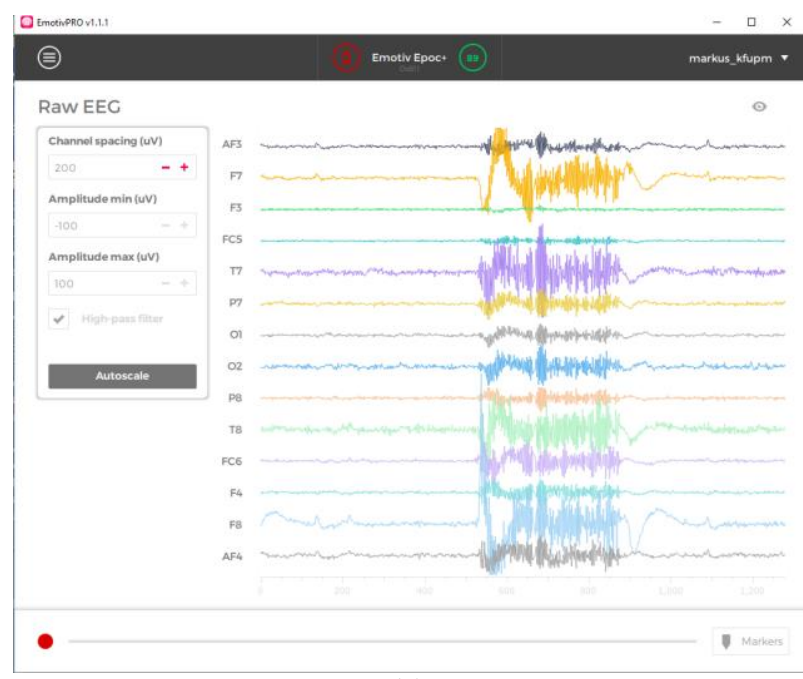

(b)

Fig. 12 raw data readings for (a) push movement and (b) clench.

We connect the system to the BCI through a client - server protocol. Moreover, we create a python script to exchange data between BCI and the system via a TCP-IP socket. The python shell running the script on the computer acted as a client while the Raspberry Pi and its circuit represents the server. The code is divided into two main parts: the client listens to a key pressed and sending it to the server through the python shell itself. Once the client sends the character, the server responds accordingly by sending the corresponding command to the Raspberry Pi, which in turn sends the signal to the infrared LED turning the TV on or OFF.

\section{Conclusion and Future Work}

In this study, a home application controlling was demonstrated using EEG-based brain-computer system. The system composed of EMOTIV EPOC headset connected to a computer that communicates to Raspberry Pi 3 platform to control a home appliance. The prototype allows home appliances to be successfully controlled by the brain electrical activity in real time. EMOTIV was used to acquire the EEG signal and communicates it to a personal computer, which is running EmoKey. The received signals are then detected and a specific character is produced accordingly. The character is then sent to the server using TCP connection and received at the other end by the Raspberry Pi, which turns ON or OFF a TV device. A python script was written to build the server that was used in transmission. One male subject participated in the experiments and he was trained to use pull the virtual cube and control his clench intensity. Simplicity of the architecture design and avoiding relying on extra hardware was at utmost importance. The results of this study demonstrated the feasibility and simplicity of our proposed BCI system to control home appliance which could be of a great aid to patients with motor disabilities.

\section{References}

[1] Maskeliunas, R., Damasevicius, R., Martisius, I., Vasiljevas, M.: Consumer grade EEG devices: are they usable for control tasks? PeerJ. 4, e1746 (2016). doi:10.7717/peerj.1746 https://doi.org/10.7717/peerj.1746

[2] EMOTIV EPOC+ - 14 Channel Wireless EEG Headset, http://www.emotiv.com/epoc/

[3] Raspberry Pi 3 Model B - Raspberry Pi, https://www.raspberrypi.org/products/raspberry-pi-3model-b/

[4] Helal, S., Mann, W., El-Zabadani, H., King, J., Kaddoura, Y., Jansen, E.: The Gator Tech Smart House: a programmable pervasive space. Computer. 38, 50-60 (2005). https://doi.org/10.1109/MC.2005.107

[5] Li, X., Xu, G., Li, L.: RFID based Smart Home architecture for improving lives. In: Security and Identification 2008 2nd International Conference on Anticounterfeiting. pp. 440-443 (2008)

[6] Aqeel-ur-Rehman, R.A., Khursheed, H.: Voice Controlled Home Automation System for the Elderly or Disabled People. Journal of Applied Environmental and Biological Sciences. 4, 55-64 (2014)

[7] Suresh, G., \& Shanmugaraju2, S. (2016, May \& june). ESense Algorithm Based Wireless Wheelchair Control ... Retrieved February 2, 2018.

[8] Alrajhi, W., Hosny, M., Al-Wabil, A., \& Alabdulkarim, A. (2014). Human Factors in the Design of BCIControlled Wheelchairs. Human-Computer Interaction. Advanced Interaction Modalities and Techniques Lecture Notes in Computer Science, 513-522. doi:10.1007/978-3319-07230-2 49 https://doi.org/10.1007/978-3-31907230-2_49 
[9] Neural-Interface-Emotiv-EPOC-and-Arduino-BrainComputer-Interaction-in-a-Proof-of-Concept.pdf, https://www.researchgate.net/profile/Vinicius_Ferreira3/p ublication/261759871_Neural_Interface_Emotiv_EPOC_ and_Arduino_Brain-

Computer_Interaction_in_a_Proof_of_Concept/links/004 635356e1a8bfc0f000000/Neural-Interface-Emotiv-EPOCand-Arduino-Brain-Computer-Interaction-in-a-Proof-ofConcept.pdf

[10] Konstantinidis, E., Conci, N., Bamparopoulos, G., Sidiropoulos, E., Natale, F. D., \& Bamidis, P. (2015). Introducing Neuroberry, a platform for pervasive EEG signaling in the IoT domain. Proceedings of the 5th EAI International Conference on Wireless Mobile Communication and Healthcare - "Transforming healthcare through innovations in mobile and wireless technologies". doi:10.4108/eai.14-10-2015.2261698 https://doi.org/10.4108/eai.14-10-2015.2261698

[11] Alshbatat, A.I.N., Vial, P.J., Premaratne, P., Tran, L.C. EEG-based brain-computer interface for automating home appliances. (2014)

[12] EEG Based Brain Controlled Robo and Home Appliancespdf.pdf

[13] Lee, W.T., Nisar, H., Malik, A.S., Yeap, K.H.: A brain computer interface for smart home control. In: 2013 IEEE International Symposium on Consumer Electronics (ISCE). pp. 35-36 (2013) https://doi.org/10.1109/ISCE.2013.6570240 